\section{Prevalência de excesso de peso e obesidade abdominal, segundo parâmetros antropométricos, e associação com maturação sexual em adolescentes escolares}

\author{
Prevalence of overweight and abdominal obesity \\ according to anthropometric parameters and the \\ association with sexual maturation in adolescent \\ schoolchildren
}

\author{
1 Universidade Federal de \\ Pernambuco, Recife, Brasil. \\ 2 Instituto Materno Infantil \\ de Pernambuco, Recife, \\ Brasil. \\ Correspondência \\ I. C. S. Pinto \\ Universidade Federal de \\ Pernambuco. \\ Rua Francisco de Barros \\ Barreto 376, apto. 1404-B, \\ Recife, PE 51021-550, Brasil. \\ isabelcspinto@yahoo.com.br
}

\begin{abstract}
The objective of the present study was to estimate the prevalence of overweight and abdominal obesity in schoolchildren according to anthropometric parameters and sexual maturation. A crosssectional study was performed in 1,405 children of both genders aged 10-14 years, living in Recife, Pernambuco State, Brazil, in 2007. Body mass index (BMI), waist circumference (WC), and waist to-height ratio (WHtR) were assessed. Sexual maturation was self-assessed. Early sexual maturation was defined as the chronological age below the median age for the referred stage. Prevalence of overweight was $20.4 \%$ (95\% CI: 18.3-22.6), and abdominal obesity was 14.9\% (95\%CI: 13.1-16.9) and 12.6\% (95\%CI: 10.9-14.4) according to WC and WHtR, respectively. There was a strong posi-

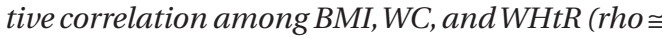
$0.8 ; p<0.001)$. In both genders, the prevalence of overweight and abdominal obesity was higher in the final stages of sexual maturation $(p<0.05)$ The high prevalence of overweight requires $u r$ gent preventive measures and control. Therefore, the inclusion of sexual maturation for the assess ment of nutritional status is recommended.
\end{abstract}

Abdominal Obesity; Sexual Maturation; Anthropometry; Students
Isabel Carolina da Silva Pinto 1,2

Ilma Kruze Grande de Arruda 1

Alcides da Silva Diniz 1

Ana Márcia Tenório de Souza Cavalcanti 1

\section{Introdução}

Nas últimas décadas, estudos vêm demonstrando um rápido e significativo aumento na prevalência mundial de obesidade, especialmente durante a infância e adolescência, tomando proporções de uma verdadeira epidemia mundial 1 .

No cenário mundial, comparando-se inquéritos populacionais realizados nos Estados Unidos da América, entre 1971-1994, observou-se um aumento de $15,4 \%$ para $25,6 \%$ na ocorrência de obesidade, na faixa etária entre 6 e 18 anos, com um crescimento relativo de $66,2 \%$ na sua prevalência. Na China, entre 1991-1997, esse aumento foi da ordem de $20,3 \%$ em crianças e adolescentes 2 , enquanto, na Inglaterra, esse acréscimo de sobrepeso foi de $44,2 \%$, entre crianças de 4-11 anos, no período de 1974-1994 ${ }^{3}$.

No Brasil, no entanto, quando se comparam os resultados do Estudo Nacional de Despesas Familiares (ENDEF), realizado em 1974-1975, e da Pesquisa sobre Padrões de Vida (PPV) realizada em 1996-1997, compreendendo apenas as regiões Sudeste e Nordeste, constatou-se um aumento de $4,1 \%$ para $13,9 \%$ na prevalência de excesso de peso, em indivíduos de 6-18 anos, correspondendo a um incremento relativo desse distúrbio de $239 \% 2$.

Dados populacionais mais recentes referentes à Pesquisa de Orçamentos Familiares (POF 2002-2003) indicam uma prevalência de excesso 
de peso nos adolescentes brasileiros na ordem de $16,7 \% 4$.

No Nordeste do Brasil, são escassos os estudos de representatividade populacional que avaliaram a ocorrência de sobrepeso/obesidade em adolescentes 5,6. Em Recife, essa escassez fica mais evidente, uma vez que inexistem estudos de base populacional abordando a questão do excesso de peso em adolescentes.

Embora a literatura já evidencie que a obesidade na infância e na adolescência pode perdurar até a fase adulta, implicando aumento do risco de problemas vasculares e metabólicos, ainda há controvérsias quanto ao método de maior validade para identificar o sobrepeso/obesidade nessa faixa etária 7 .

O índice de massa corporal (IMC) tem sido o método antropométrico mais utilizado para avaliação do estado nutricional, por ser uma medida simples e de baixo custo e que tem apresentado uma boa concordância entre os indicadores de adiposidade no diagnóstico de sobrepeso e obesidade 8 e o risco de doença cardiovascular 9 . Apesar dessas vantagens, os inúmeros pontos de discriminação diagnóstica do IMC, embasados em estudos empíricos nacionais 10 ou internacionais 11,12 , têm dificultado, sobremaneira, a construção de uma base de referência que permita uma estimativa mais consistente da magnitude $\mathrm{e}$ evolução desse distúrbio nutricional.

A circunferência da cintura (CC) e a razão cintura-estatura (RCEst) têm sido propostas como medidas antropométricas adicionais para avaliar a obesidade e adiposidade central porque estariam associadas ao impacto adverso nos fatores de risco cardiovascular, independentemente da condição do peso corporal, sendo benéfico para identificação precoce de crianças que se beneficiariam com uma intervenção precoce ${ }^{13}$. Estudos demonstram que esses parâmetros seriam melhores preditores de risco cardiovascular em crianças e adolescentes quando comparados ao IMC isoladamente 14,15,16.

Na adolescência, além dos parâmetros antropométricos e de composição corporal é de fundamental importância considerar não apenas a idade cronológica, mas também o estágio de maturação sexual 17. Alguns estudos vêm demonstrando a associação entre a maturação sexual precoce e obesidade em adolescentes, particularmente no sexo feminino 18,19 .

O objetivo deste estudo foi estimar a prevalência de excesso de peso e obesidade abdominal em adolescentes escolares segundo parâmetros antropométricos e de maturação sexual.

\section{Metodologia}

Estudo de corte transversal, envolvendo adolescentes de 10-14 anos, de ambos os sexos, matriculados em escolas da rede pública e privada do Município do Recife, Pernambuco, Brasil, realizado no período de outubro/dezembro de 2007. A população elegível incluiu todos os adolescentes presentes no âmbito escolar por ocasião da coleta dos dados e excluídos aqueles que apresentassem qualquer deficiência física que comprometesse a avaliação antropométrica.

O cálculo para estimar o tamanho da amostra baseou-se em uma prevalência estimada (p) de excesso de peso de $19,5 \% 5$, um erro amostral (d) de 3\% e um nível de 95\% de confiança (z), utilizando-se a fórmula $\mathrm{n}=\left[\mathrm{z}^{2} \mathrm{p} .(1-\mathrm{p})\right] / \mathrm{d}^{2} 20$. Como o processo de seleção da amostra foi do tipo polietapas, cujas unidades amostrais foram a escola (1o conglomerado), o turno (2o conglomerado), a turma (3o conglomerado) e o escolar (4o conglomerado), o "n" amostral foi ajustado pelo efeito do desenho do estudo, mediante o uso de um fator de correção da ordem de 2,1 20 totalizando um número mínimo de 1.403 escolares. Para corrigir eventuais perdas ou recusas, esse valor foi acrescido em $5 \%$, utilizando a fórmula [100/ (100-n)], no qual "n" representa o valor percentual, perfazendo uma amostra em torno de 1.477 escolares.

Para a seleção da amostra, procedeu-se a um levantamento do número total de escolas públicas e privadas que ofereciam de $5 \underline{a}$ a $8 \underline{a}$ séries do ensino fundamental na cidade do Recife, no ano de 2007, totalizando 578 e 352 escolas. respectivamente, visando imprimir a proporcionalidade necessária numa amostra do tipo estratificada. Do total foram selecionadas por sorteio aleatório 40 escolas, sendo 29 públicas e 11 privadas. Na segunda e na terceira etapas, foram selecionados de forma aleatória simples o turno e a turma de cada escola. Posteriormente, foram selecionados por sorteio no máximo 40 alunos por escola mediante a utilização de uma tabela de números aleatórios.

A coleta dos dados foi feita por uma equipe de técnicos previamente treinada para aplicação do questionário e aferição das medidas antropométricas (peso, altura e circunferência da cintura), seguidas às recomendações de Lohman et al. 21 . Antes da coleta dos dados, todos os avaliadores da equipe foram treinados e adequadamente testados quanto à precisão na aferição das medidas, tomando-se como padrão de referência um antropometrista especialista em avaliação antropométrica, treinado segundo as normas vigentes, visando testar a reprodutibilidade interavaliadores. Por sua vez, a avaliação intra-avaliador foi 
testada pela aferição das medidas repetidas pelo mesmo avaliador para testar a confiabilidade das medidas. Apenas aqueles antropometristas que apresentaram um escore para o índice kappa > 0,8 foram aceitos para participar da coleta das medidas antropométricas.

O peso foi aferido em balança digital eletrônica, da marca Plenna-MEA-03140 (Plenna, São Paulo, Brasil), com capacidade de até $150 \mathrm{~kg}$ e precisão de 100g. Os adolescentes foram pesados descalços, sem objetos nas mãos e nos bolsos e sem adornos na cabeça. A altura foi determinada com fita métrica Stanley milimetrada, com precisão de $1 \mathrm{~mm}$ e exatidão de $0,5 \mathrm{~cm}$. A fita foi afixada na parede e os adolescentes colocados em posição ereta, descalços, com os membros superiores pendentes ao longo do corpo, os calcanhares, o dorso e a cabeça tocando a parede, e olhando para frente 21 .

As medidas de peso e estatura foram utilizadas para cálculo do IMC [peso $(\mathrm{kg})$ /estatura $(\mathrm{m})^{2}$ ]. Para o diagnóstico de sobrepeso e obesidade foram adotados os pontos de corte propostos por Cole et al. ${ }^{12}$ que propõe valores de corte do IMC equivalentes na adolescência aos valores de corte do IMC tradicionalmente utilizados para diagnosticar o excesso de peso e a obesidade em adultos, sendo equivalentes ao percentil da distribuição do IMC que corresponda, na idade de 18 anos, ao IMC de $25 \mathrm{~kg} / \mathrm{m}^{2}$ e $30 \mathrm{~kg} / \mathrm{m}^{2}$, respectivamente. Para análise da nossa casuística, os indivíduos com sobrepeso foram agrupados aos obesos, sendo classificados na categoria de excesso de peso.

A CC foi mensurada por meio de uma fita métrica inextensível, com o adolescente em pé com abdômen relaxado, os braços descontraídos ao lado do corpo, sendo a fita colocada horizontalmente no ponto médio entre a borda inferior da última costela e a crista ilíaca 21 . Foram adotados os pontos de corte propostos por Taylor et al. 22, que identifica obesidade abdominal quando $C C \geq \mathrm{P}_{80}$. A RCEst foi obtida pela razão entre a circunferência da cintura $(\mathrm{cm}) \mathrm{e}$ a altura $(\mathrm{cm})$, estabelecendo-se como ponto de corte para obesidade abdominal valores iguais ou superiores a 0,5 16,23.

As medidas antropométricas aferidas em duplicata pelo mesmo avaliador foram desprezadas quando o erro de aferição entre elas foi maior que $100 \mathrm{~g}$ para peso, $0,5 \mathrm{~cm}$ para altura e $0,1 \mathrm{~cm}$ para circunferência da cintura. $O$ valor resultante das aferições foi a média entre elas.

A avaliação da maturação sexual foi feita por intermédio da auto-avaliação baseada em 5 estágios propostos por Tanner 24 , sendo o estágio 1 correspondente sempre à fase infantil, impúbere, e o estágio 5 à fase pós-puberal, adulta. O estádio da maturação sexual foi feito pela avaliação das mamas no sexo feminino e da genitália externa no sexo masculino. As mamas e os genitais foram avaliados quanto ao tamanho, forma e características. Para definir como maturação sexual precoce foi tomada a mediana de idade da população estudada para cada estágio de maturação, de acordo com o sexo (Tabela 1). Definiu-se como maturação sexual precoce o indivíduo que apresentasse idade cronológica inferior à mediana de idade para o estágio de maturação auto-referido pelo escolar 19 .

Os dados foram digitados em dupla entrada e verificados com o "validate", módulo do programa Epi Info, versão 6.04 (Centers for Disease Control and Prevention, Atlanta, Estados Unidos), para identificar eventuais inconsistências. Os dados foram analisados com o auxílio do programa SPSS versão 13.0 (SPSS Inc., Chicago, Estados Unidos). Na descrição das proporções, procedeu-se a uma aproximação da distribuição binomial à distribuição normal pelo intervalo de 95\% de confiança (IC95\%). Na comparação das proporções, utilizou-se o teste do qui-quadrado de Pearson e/ou com tendência linear, quando aplicável. As variáveis contínuas foram testadas quanto à normalidade da distribuição pelo teste de Kolmogorov Smirnov. As variáveis IMC, CC e RCEst apresentaram distribuição não normal, mesmo após transformação em logaritmo natural e foram expressas sob a forma de mediana e intervalo interquartílico. Para análise das correlações, utilizou-se o teste de Spearman, e para comparação entre medianas de amostras independentes, usou-se o teste U de MannWhitney. O nível de significância adotado foi de $5 \%$.

O protocolo de pesquisa foi avaliado e aprovado pelo Comitê de Ética em Pesquisa, Centro de Ciências da Saúde, Universidade Federal de Pernambuco (CEP/CCS/UFPE), sob o $n^{\circ}$.

Tabela 1

Distribuição das medianas de idade para cada estágio de maturação sexual, segundo o sexo, em escolares de 10-14 anos. Recife, Pernambuco, Brasil, 2007.

\begin{tabular}{lcc}
\hline $\begin{array}{l}\text { Estágio de maturação } \\
\text { sexual }\end{array}$ & \multicolumn{2}{c}{ Mediana de idade (anos) } \\
& Masculino & Feminino \\
\hline 1 & 10 & 10 \\
2 & 11 & 10 \\
3 & 12 & 11 \\
4 & 13 & 13 \\
5 & 13 & 13
\end{tabular}


170/2006, de acordo com a Resolução no . 196/1096 do Conselho Nacional de Saúde. Os adolescentes que aceitaram participar do estudo foram previamente informados dos objetivos da pesquisa bem como os métodos a serem adotados e o pai ou responsável assinou um termo de compromisso livre e esclarecido.

\section{Resultados}

Foram entrevistados 1.477 adolescentes dos quais foram descartados os questionários de 72 sujeitos por não obedeceram à reprodutibilidade estabelecida para aferição das medidas antropométricas, totalizando 1.405 adolescentes elegíveis para fins desse estudo. Desses, 16 (2,6\%) meninos e $22(2,8 \%)$ meninas não responderam à auto-avaliação para maturação sexual. Do total da amostra, $605(43,1 \%)$ eram do sexo masculino e $800(56,9 \%)$ do sexo feminino. A mediana de idade foi de 12 anos (IQ 11-13).

A prevalência de sobrepeso foi de 15,9\% (IC95\%: 14,00-17,89) e obesidade de 4,5\% (IC95\%: 3,53-5,78), totalizando 20,4\% (IC95\%: 18,3-22,6) de adolescentes com excesso de peso. A obesidade abdominal foi diagnosticada em 14,9\% (IC95\%: 13,1-16,9) e 12,6\% (IC95\%: 10,9-14,4) dos escolares, utilizando os parâmetros CC e RCEst, respectivamente.

A prevalência de excesso de peso foi maior quando comparada à prevalência de obesidade abdominal. Não houve diferença na distribuição de excesso de peso e obesidade abdominal na desagregação por sexo. Todavia, a prevalência de excesso de peso apresentou diminuição com o aumento progressivo da idade ( $p=0,003)$, o que não ocorreu com os indicadores de obesidade abdominal (CC e RCEst) ( $\mathrm{p}>0,05)$ (Tabela 2).

Quando avaliado o "pool” de indicadores antropométricos, 10,9\% (IC95\%: 9,30-12,64) da população estudada, apresentaram concomitantemente IMC $\geq \mathrm{P}_{85}, \mathrm{CC} \geq \mathrm{P}_{80}$ e RCEst $\geq 0,5$.

Os indicadores IMC, CC e RCEst apresentaram uma forte correlação positiva entre si (rho 0,8; $<<0,001$ ) (Figura 1).

As prevalências de excesso de peso e obesidade abdominal mostraram um incremento $(\mathrm{p}<$ $0,05)$ nos estágios finais da maturação sexual, para ambos os sexos, quando avaliados os indicadores IMC e CC (Tabela 3).

Quando os adolescentes foram avaliados segundo o sexo e o grau de maturação sexual (precoce e normal/tardio), as meninas com maturação sexual precoce apresentaram maior $(\mathrm{p}=$ 0,000 ) prevalência de excesso de peso (IMC $\geq P_{85}$ ) e obesidade abdominal $\left(\mathrm{CC} \geq \mathrm{P}_{80}\right.$ ) do que as adolescentes que apresentaram grau de maturação sexual normal/tardio, ressaltando-se que a prevalência de obesidade abdominal (RCEst $\geq 0,5$ ) também mostrou uma tendência mais elevada entre as que maturaram mais cedo, embora sem a devida significância estatística $(p=0,060)$. Nos meninos, apenas o excesso de peso (IMC $\geq \mathrm{P}_{85}$ ) apresentou-se maior ( $\mathrm{p}=0,020$ ) naqueles que apresentaram maturação sexual precoce quando comparado aos que apresentavam grau de maturação normal/tardio (Figura 2).

Comparando-se os indicadores IMC, CC e RCEst, enquanto variáveis contínuas, as adolescentes do sexo feminino que maturaram precocemente apresentaram medianas maiores $(\mathrm{p}<$ $0,05)$ para todos os indicadores avaliados quando comparadas as que apresentaram maturação sexual normal/tardia. Porém, não foi observado comportamento similar para o sexo masculino no que diz respeito aos indicadores IMC ( $\mathrm{p}=$ $0,302$ ) e RCEst ( $p=0,104)$. Em contrapartida, a mediana de CC no sexo masculino foi menor ( $\mathrm{p}=$ 0,026 ) naqueles que apresentaram maturação sexual precoce.

\section{Discussão}

Estudos populacionais têm mostrado que a obesidade em crianças e adolescentes brasileiros vem crescendo drasticamente, tendo triplicado entre os anos de 1975-1997 2. Confrontando-se a prevalência $(20,4 \%)$ verificada na nossa casuística com dados da PPV (11\%), realizada no Brasil em 1997 25, encontra-se um incremento extremamente elevado (185\%) na prevalência de excesso de peso em adolescentes da região urbana no Nordeste do Brasil. Tal aumento vem sendo observado em outras regiões do Brasil, ainda que sejam escassos os estudos de representatividade populacional que utilizaram a mesma faixa etária e os pontos de corte para diagnóstico do excesso de peso.

A prevalência de excesso de peso encontrada em nossa casuística foi similar àquela descrita em outros espaços geográficos, com os mesmos pontos de corte utilizado neste estudo. Suñé et al. 26, em estudo de base escolar envolvendo adolescentes de Capão de Canoa, cidade do Sul do Brasil, relataram prevalência de sobrepeso ou obesidade de $24,8 \%$. Por sua vez, Terres et al. 27 encontraram resultados similares (25,9\%) em adolescentes escolares maiores de 14 anos na cidade de Pelotas, Rio Grande do Sul, também na Região Sul do Brasil, embora Farias Júnior \& Silva 6 tenham encontraram prevalências de $10 \%$ em adolescentes residentes da cidade João Pessoa, Paraíba. Utilizando os pontos de corte propostos por Must et al. 11, Campos et 
Prevalência de excesso de peso e obesidade abdominal, segundo faixa etária, em escolares de 10-14 anos. Recife, Pernambuco, Brasil, 2007.

\begin{tabular}{|c|c|c|c|c|c|c|c|}
\hline \multirow[t]{3}{*}{ Idade (anos) } & \multirow[t]{3}{*}{$\mathbf{n}$} & \multirow{2}{*}{\multicolumn{2}{|c|}{$\begin{array}{l}\text { Excesso de peso } \\
\text { IMC }\end{array}$}} & \multicolumn{4}{|c|}{ Obesidade Abdominal } \\
\hline & & & & \multicolumn{2}{|c|}{$\mathrm{CC}$} & \multicolumn{2}{|c|}{ RCEst } \\
\hline & & $\%$ & IC95\% & $\%$ & IC95\% & $\%$ & IC95\% \\
\hline 10 & 275 & 23,6 & $18,7-29,1$ & 13,8 & $10,0-18,5$ & 10,5 & $7,2-14,8$ \\
\hline 11 & 363 & 20,9 & $16,9-25-5$ & 16,5 & $12,9-20,8$ & 15,2 & $11,6-19,3$ \\
\hline 12 & 309 & 25,6 & $20,8-30,8$ & 19,4 & $15,2-24,3$ & 15,5 & $11,7-20,1$ \\
\hline 13 & 283 & 15,2 & $11,2-20,0$ & 11,3 & $7,9-15,6$ & 8,8 & $5,8-12,8$ \\
\hline 14 & 175 & 13,7 & $9,0-19,7$ & 11,4 & $7,1-17,1$ & 11,4 & $7,1-17,1$ \\
\hline Total & 1.405 & 20,4 & $18,3-22,6$ & 14,9 & $13,1-16,9$ & 12,6 & $10,9-14,4$ \\
\hline Valor de $\mathrm{p}^{*}$ & & & 0,003 & & 0,200 & & 0,430 \\
\hline
\end{tabular}

IMC: índice de massa corporal; CC: circunferência da cintura; RCEst: razão cintura-estatura; IC95\%: intervalo de 95\% de confiança.

* Qui-quadrado de tendência linear.

al. 5 encontraram excesso de peso em 19,5\% dos adolescentes com idade entre 10 e 19 anos na cidade de Fortaleza, Ceará.

No Recife, não há estudos de abrangência populacional que tenham avaliado a prevalência de excesso de peso em adolescentes. Contudo, Silva et al. 28 , estudando uma amostra de crianças e adolescentes de diferentes classes sócio-econômicas, selecionada por conveniência, encontraram uma prevalência de sobrepeso e/ou obesidade de 22,8\%, semelhante a nossa casuística.

Não foi verificada diferença entre as prevalências de excesso de peso na desagregação por sexo. Entretanto, houve uma tendência na diminuição do número dos adolescentes com sobrepeso ou obesidade pari passu ao aumento progressivo da idade, semelhante ao encontrado em outros estudos 5,26. Tal fato poderia ser atribuído à maior preocupação com a aparência física e, portanto, com o excesso de peso em adolescentes com idade mais avançada.

Dentre as ferramentas para avaliação do estado nutricional em adolescentes, o IMC é o método antropométrico mais utilizado, tanto em serviços de saúde como em estudos populacionais para rastrear o sobrepeso e a obesidade. A importância desse indicador se deve ao fato de correlacionar-se com a adiposidade, aumento dos níveis pressóricos e lipídeos séricos, sendo considerado como preditor do risco cardiovascular em adultos 7. Mas as implicações de elevados valores de IMC na vida futura de crianças e adolescentes são pouco claras 29. O IMC isoladamente parece não refletir as grandes mudanças na composição corporal que ocorrem durante a adolescência, levando em consideração o estágio de maturação sexual e o maior acúmulo de gordura nas meninas e aumento da massa magra nos meninos 13 .

Os resultados encontrados em nossa casuística apontam para uma forte correlação entre os indicadores avaliados (IMC, CC e RCEst), o que também tem sido descrito por outros autores 8,30 . Por mais que tenham apresentado uma forte correlação, o uso de mais de um indicador parece apontar informações adicionais, sobretudo no que diz respeito ao risco de comorbidades relacionadas ao excesso de peso quando associado também à adiposidade central. Nesse sentido, Janssen et al. 31 demonstraram que entre os adolescentes com sobrepeso, diagnosticado pelo IMC, aqueles que apresentaram elevação concomitante da CC estariam sob risco duas vezes maior para aumento de triglicerídeos, insulina e síndrome metabólica, quando comparados ao grupo de sobrepeso sem obesidade abdominal. Destarte, a utilização da CC associada ao IMC para o diagnóstico da obesidade em adolescentes e triagem dos pacientes de risco de morbidades, sobretudo cardiovasculares, tem sido fortemente recomendada.

Os pontos de corte para avaliação da CC propostos por Taylor et al. 22 foram estabelecidos utilizando como padrão-ouro para avaliação da adiposidade o dual energy $X$-ray absorptiometry (DEXA), no qual o percentil 80 apresentou maior sensibilidade e especificidade para detecção de acúmulo de massa gorda na região do tronco, tanto em meninas quanto em meninos, na faixa etária entre 2 a 19 anos. A utilização dos pontos 
Figura 1

Curvas de correlação entre índice de massa corporal (IMC), circunferência da cintura (CC) e razão cintura-estatura (RCEst), em escolares de 10-14 anos. Recife, Pernambuco, Brasil, 2007.

1a) Correlação CC e IMC

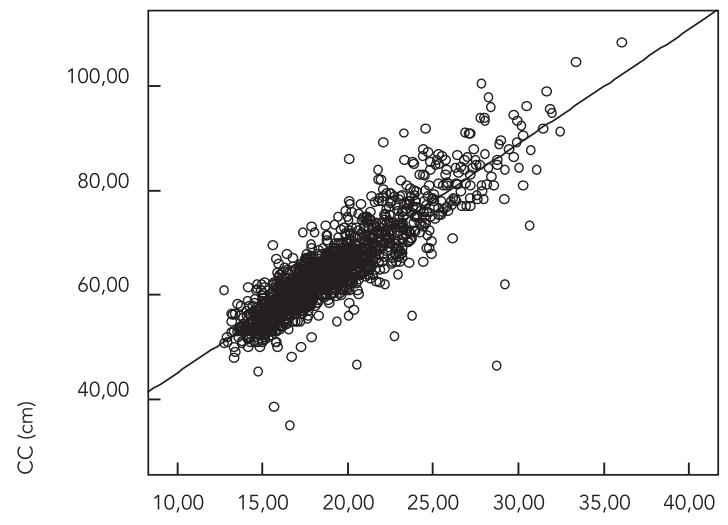

rho $=0,89$

$p<0,001$

IMC $\left(\mathrm{kg} / \mathrm{m}^{2}\right)$

1b) Correlação RCEst e IMC

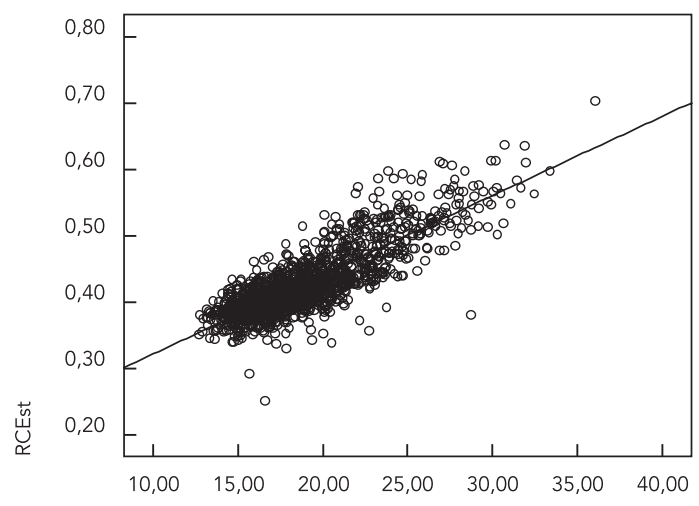

rho $=0,79$

$p<0,001$

$\operatorname{IMC}\left(\mathrm{kg} / \mathrm{m}^{2}\right)$

1c) Correlação RCEst e CC

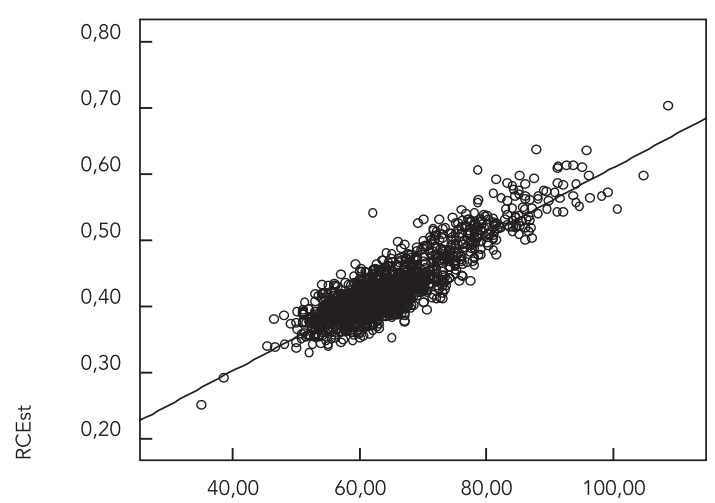

rho $=0,82$

$p<0,001$

$\mathrm{CC}(\mathrm{cm})$ 
Tabela 3

Prevalência de excesso de peso e obesidade abdominal, segundo o estágio de maturação sexual e o sexo, em escolares de 10-14 anos. Recife, Pernambuco, Brasil, 2007.

\begin{tabular}{|c|c|c|c|c|c|c|c|c|}
\hline \multirow{3}{*}{$\begin{array}{l}\text { Estágio de } \\
\text { maturação sexual }\end{array}$} & \multicolumn{4}{|c|}{ Masculino } & \multicolumn{4}{|c|}{ Feminino } \\
\hline & \multirow[t]{2}{*}{$n$} & \multirow{2}{*}{$\begin{array}{l}\text { Excesso } \\
\text { de peso } \\
\text { IMC (\%) }\end{array}$} & \multicolumn{2}{|c|}{$\begin{array}{l}\text { Obesidade } \\
\text { abdominal }\end{array}$} & \multirow[t]{2}{*}{$n$} & \multirow{2}{*}{$\begin{array}{l}\text { Excesso } \\
\text { de peso } \\
\text { IMC (\%) }\end{array}$} & \multicolumn{2}{|c|}{$\begin{array}{l}\text { Obesidade } \\
\text { abdominal }\end{array}$} \\
\hline & & & CC (\%) & RCEst (\%) & & & CC (\%) & RCEst (\%) \\
\hline 1 & 58 & 15,5 & 10,3 & 10,3 & 47 & 8,5 & 6,4 & 6,4 \\
\hline 2 & 160 & 16,3 & 10,0 & 12,5 & 86 & 18,6 & 12,8 & 9,3 \\
\hline 3 & 183 & 18,6 & 15,8 & 14,2 & 251 & 17,9 & 12,0 & 10,8 \\
\hline 4 & 134 & 26,9 & 24,6 & 20,1 & 341 & 23,5 & 17,9 & 11,7 \\
\hline 5 & 54 & 22,2 & 16,7 & 13,0 & 53 & 32,1 & 17,0 & 17,0 \\
\hline Total & 589 & 19,9 & 15,8 & 14,6 & 778 & 20,8 & 14,7 & 11,2 \\
\hline Valor de $p$ * & & 0,036 & 0,018 & 0,146 & & 0,002 & 0,017 & 0,098 \\
\hline
\end{tabular}

IMC: índice de massa corporal; CC: circunferência da cintura; RCEst: razão cintura-estatura; IC95\%: intervalo de $95 \%$ de confiança.

* Qui-quadrado de tendência linear.

Figura 2

Prevalência de excesso de peso e obesidade abdominal, segundo o grau de maturação sexual e o sexo, em escolares de 10-14 anos. Recife, Pernambuco, Brasil, 2007.

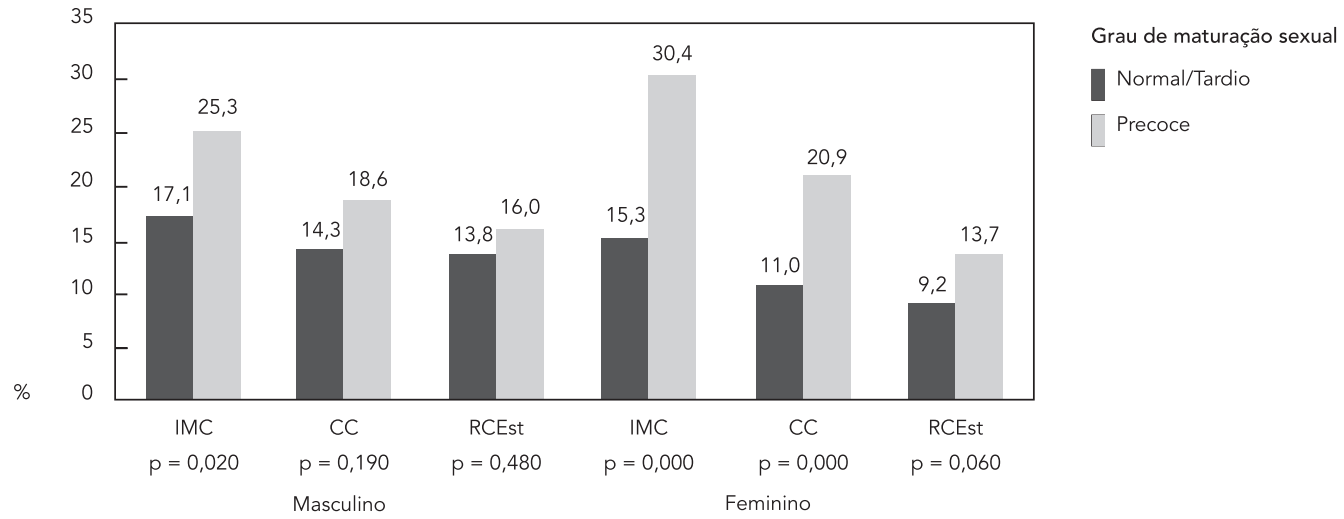

IMC: índice de massa corporal; CC: circunferência da cintura; RCEst: razão cintura-estatura.

Nota: teste de qui-quadrado de Pearson.

de corte propostos por estes autores para avaliação da obesidade abdominal na população do presente estudo foi embasada na maior sensibilidade apresentada para detecção das alterações de IMC, concentrações séricas de colesterol total, glicemia, insulina e leptina, em crianças e adolescentes, sendo indicado em estudos de saúde pública como melhor ponto de discrimina- ção diagnóstica de tais distúrbios 32 . Em adição, Sant'Anna et al. 33, ao estudar qual o melhor sítio anatômico para avaliar a circunferência da cintura em crianças, concluíram que o ponto médio entre a borda inferior da última costela e a crista ilíaca apresentou a maior correlação com o percentual de gordura do corpo. 
Vale salientar que ainda são escassos os estudos sobre avaliação da circunferência da cintura em crianças e adolescentes, bem como não existem pontos de corte específicos para nossa população, levando-nos a utilizar curvas referentes a outras populações, fato que pode incorrer em erros de estimação para a real prevalência da obesidade abdominal.

Outro parâmetro que vem sendo utilizado mais recentemente na avaliação da obesidade abdominal é RCEst. Estudos conduzidos nos Estados Unidos 15, na Inglaterra 16 e na Austrália 34, todos envolvendo crianças e adolescentes, concluíram que a utilização da RCEst seria o melhor preditor para risco cardiovascular, em detrimento do uso do IMC isoladamente, além de oferecer a vantagem de ser calculado facilmente, não precisar de pontos de corte específicos para idade e sexo e poder transmitir a mensagem para profissionais e familiares que o ideal é manter a CC menor que a metade da altura.

A avaliação do estado nutricional na adolescência se torna complexa porque a idade cronológica perde parte de sua importância nessa fase. Poucos são os estudos que relacionam o estado nutricional e o grau de maturação sexual, sendo este, na maioria das vezes, estudado pela presença da menarca nas meninas 35,36 e pela primeira ejaculação ou presença de pêlos axilares, nos meninos, quando estes são avaliados 27

Na nossa casuística, houve uma maior prevalência de sobrepeso ou obesidade nos estágios finais da maturação sexual, especialmente no sexo feminino. Esse achado aparentemente apresenta uma contradição quando comparado à diminuição da prevalência de excesso de peso com o aumento da idade. Tal fato pode ser explicado pela influência do estádio puberal nos parâmetros antropométricos e de composição corporal, que, quando não levado em consideração, pode resultar em interpretação não fidedigna do estado nutricional quando este é avaliado, tomando-se como parâmetro apenas a idade 17 .

É durante a puberdade que ocorrem as alterações hormonais responsáveis pelo aparecimento dos caracteres secundários e as transformações físicas. A idade cronológica, por si só, não pode predizer o grau de desenvolvimento da puberdade em que o adolescente se encontra, já que este sofre influência tanto de fatores intrínsecos como ambientais. Portanto, atraso ou precocidade no início deste desenvolvimento pode resultar em alterações no crescimento e na composição corporal do adolescente 17 .

Oliveira \& Veiga 37 também observaram um maior aumento de excesso de peso nas adoles- centes nos estágios finais da maturação sexual, estando relacionado às modificações do tecido adiposo, que apresentam aumento gradual da gordura corporal durante toda a adolescência ocorrendo o inverso no sexo masculino, no qual há uma perda da gordura corporal em detrimento de um maior estoque de massa muscular.

Castilho et al. 38, estudando adolescentes brasileiros, verificaram que há aumento de IMC em ambos os sexos nos estágios finais da maturação sexual. Nas meninas há um aumento proporcional entre a massa gorda e a massa livre de gordura, enquanto no sexo masculino percebe-se um aumento da massa livre de gordura e uma redução da massa gorda, corroborando os achados da literatura.

Estudo envolvendo adolescentes americanos, com metodologia similar ao presente trabalho, verificou que meninas que apresentavam maturação sexual precoce tinham risco duas vezes maior para o desenvolvimento de obesidade, sendo o peso corporal, o IMC, a prega cutânea tricipital (PCT) e a soma das pregas cutâneas mais elevadas nesse grupo. Semelhante aos nossos resultados, os meninos não apresentaram associação entre maturação sexual precoce e indicadores antropométricos para obesidade 19 .

É importante ressaltar que a auto-avaliação da maturação sexual pode resultar em possíveis limitações para a fidedignidade do estádio puberal, embora a literatura tenha mostrado que a utilização desses dados é confiável no diagnóstico da maturação sexual 39 .

A prevalência de excesso de peso em adolescentes escolares da cidade do Recife foi alta e vem apoiar evidências de que a adolescência é um dos períodos de maior risco para o desenvolvimento da obesidade. Isso é um fato preocupante, tendo em vista a maior possibilidade de esse distúrbio nutricional permanecer durante a idade adulta, e com ele maior risco de ocorrência de morbidades.

A forte correlação observada entre os parâmetros antropométricos avaliados (IMC, CC e RCEst) vem demonstrar que qualquer um desses indicadores poderia ser utilizado em rastreamentos populacionais para detectar alterações de peso corporal. No entanto, a literatura vem mostrando que a utilização concomitante do indicador de obesidade abdominal, além do excesso de peso, seria o melhor preditor para risco cardiovascular.

Por sua vez, além dos indicadores antropométricos, é imprescindível levar em consideração o grau de maturação sexual dos adolescentes, particularmente do sexo feminino, cuja maturação sexual precoce esteve associada ao aumento da prevalência de excesso de peso se- 
gundo os indicadores antropométricos estudados. É notória a necessidade de melhorar os métodos de avaliação nutricional nesse período da vida, incluindo a avaliação da maturação sexual no diagnóstico do estado nutricional, dado que a obesidade vem assumindo proporções cada vez maiores, situação que impõe a pronta adoção de medidas de prevenção e controle.

\section{Resumo}

Este estudo objetivou estimar a prevalência de excesso de peso e obesidade abdominal em escolares segundo antropometria e maturação sexual. Foi um estudo transversal, incluindo 1.405 escolares de 10-14 anos, de ambos os sexos, residentes no Recife, Pernambuco, Brasil, em 2007. Foi avaliado o índice de massa corporal (IMC), a circunferência da cintura (CC) e razão da cintura-estatura (RCEst). A maturação sexual foi autoavaliada, definindo-se como precoce quando o escolar apresentava idade cronológica inferior à mediana de idade para o referido estágio. A prevalência de excesso de peso foi de 20,4\% (IC95\%: 18,3-22,6) e a de obesidade abdominal foi de 14,9\% (IC95\%: 13,1-16,9) para CC e 12,6\% (IC95\%: 10,9-14,4) para RCE. O IMC, a CC e a RCEst apresentaram uma forte correlação positiva (rho $\cong 0,8 ; p<0,001)$. A prevalência de excesso de peso $e$ de obesidade abdominal foi maior $(p<0,05)$ nos es tágios finais de maturação sexual em ambos os sexos. A alta prevalência de excesso de peso requer medidas urgentes de prevenção e controle desse distúrbio, sendo recomendada a inclusão da maturação sexual na avaliação do estado nutricional.

Obesidade Abdominal; Maturidade Sexual; Antropometria; Estudantes

\section{Colaboradores}

I. C. S. Pinto revisou a literatura, realizou a análise dos dados e escreveu a versão preliminar do manuscrito. I. K. G. Arruda e A. S. Diniz contribuíram com as análises dos dados e revisaram a versão final do manuscrito. A. M. T. S. Cavalcanti colaborou na elaboração do manuscrito.

\section{Agradecimentos}

Os autores agradecem o apoio financeiro ao Conselho Nacional de Desenvolvimento Científico e Tecnológico (CNPq, processo no. 444146/2006-5), ao Ministério da Saúde (processo no. 137/2006) e ao Ministério da Ciência e Tecnologia (processo $n^{\circ}$. 01.0265.00/2005). Agradecem também aos escolares pela participação como sujeitos da pesquisa, à Secretaria Municipal de Educação e às Direções das escolas pelo apoio logístico. 


\section{Referências}

1. James PT. Obesity: the worldwide epidemic. Clin Dermatol 2004; 22:276-80.

2. Wang Y, Monteiro C, Popkin BM. Trends of obesity and underweight in older children and adolescents in the United States, Brazil, China, and Russia. Am J Clin Nutr 2002; 75:971-7.

3. Chinn S, Rona RJ. Prevalence and trends in overweight and obesity in three cross sectional studies of British children, 1974-94. BMJ 2001; 322:24-6.

4. Instituto Brasileiro de Geografia e Estatística. Pesquisa de orçamentos familiares 2002-2003: antropometria e análise do estado nutricional de crianças e adolescentes no Brasil. Rio de Janeiro: Instituto Brasileiro de Geografia e Estatística; 2006.

5. Campos LA, Leite AJM, Almeida PC. Prevalência de sobrepeso e obesidade em adolescentes escolares do município de Fortaleza, Brasil. Rev Bras Saúde Matern Infant 2007; 7:183-90.

6. Farias Júnior JC, Silva KS. Sobrepeso/obesidade em adolescentes escolares da cidade de João Pessoa-PB: prevalência e associação com fatores demográficos e socioeconômicos. Rev Bras Med Esporte 2008; 14:104-8.

7. Tomkins A. Measuring obesity in children: what standards to use? J Pediatr (Rio J.) 2006; 82:246-8.

8. Giugliano R, Melo ALP. Diagnóstico de sobrepeso e obesidade em escolares: utilização do índice de massa corporal segundo padrão internacional. J Pediatr (Rio J.) 2004; 80:129:34.

9. Garnett SP, Baur LA, Srinivasan S, Lee JW, Cowell CT. Body mass index and waist circumference in midchilhood and adverse cardiovascular disease risk clustering in adolescence. Am J Clin Nutr 2007; 86:549-55.

10. Conde WL, Monteiro CA. Body mass index cutoff points for evaluation of nutritional status in Brazilian children and adolescents. J Pediatr (Rio J.) 2006; 82:266-72.

11. Must A, Dallal GE, Dietz WH. Reference data for obesity: 85th and 95th percentiles of body mass index (wt/ht2) and tríceps skinfold thickness. Am J Clin Nutr 1991; 53:839-46.

12. Cole TJ, Bellizzi MC, Flegal KM, Dietz WH. Establishing a standard definition for child overweight and obesity worldwide: international survey. BM] 2000; 320:1240-2.

13. Must A, Hollander SA, Economos CD. Childhood obesity: a growing public health concern. Expert Rev Endocrinol Metab 2006; 1:233-54.

14. Savva SC, Tornaristis M, Savva ME, Kourides Y, Panagi A, Silikiotou N, et al. Waist circumference and waist-to-height ratio are better predictors of cardiovascular disease risk factors in children than body mass index. Int J Obes Relat Metab Disord 2000; $24: 1453-8$

15. Kahn HS, Imperatore G, Cheng YJ. A populationbased comparison of BMI percentiles and waistto-height ratio for identifying cardiovascular risk in youth. J Pediatr 2005; 146:482-8.
16. McCarthy HD, Ashwell M. A study of central fatness using waist-to-height ratios in UK children and adolescents over two decades supports the simple message - 'keep your waist circumference to less than half your height'. Int J Obes Relat Metab Disord 2006; 30:988-92.

17. Barbosa KBF, Franceschini SCC, Priore SE. Influência dos estágios de maturação sexual no estado nutricional, antropometria e composição corporal de adolescentes. Rev Bras Saúde Matern Infant 2006; 6:375-82.

18. Bini V, Celi F, Berioli MG, Bacosi ML, Stella P, Tosti L, et al. Body mass index in children and adolescents according to age and pubertal stage. Eur J Clin Nutr 2000; 54:214-8.

19. Wang Y. Is obesity associated with early sexual maturation? A comparison of the association in American boys versus girls. Pediatrics 2002; 110:903-10.

20. Henderson RH, Sundaresan T. Cluster sampling to assess immunisation coverage: a review of experience with a simplified sampling method. Bull World Health Organ 1992; 60:253-60.

21. Lohman TG, Roche AF, Martorell R. Anthropometric standardization reference manual. Champaign: Human Kinetics Books; 1988.

22. Taylor RW, Jones IE, Williams SM, Goulding A. Evaluation of waist circumference, waist-to-hip ratio, and the conicity index as screening tools for high trunk fat mass, as measured by dual-energy X-ray absorptiometry, in children 3-19y. Am J Clin Nutr 2000; 72:490-5.

23. Li C, Ford ES, Mokdad AH, Cook S. Recent trends in waist circumference and waist-height ratio among US children and adolescents. Pediatrics 2006; 118:1390-8.

24. Tanner JM. Growth at adolescence. $2^{\text {nd }}$ Ed. Oxford Blackwell Scientific; 1962.

25. Veiga GV, Cunha AS, Sichieri R. Trends in overweight among adolescents living in poorest and richest regions of Brazil. Am J Public Health 2004 94:1544-8.

26. Suñé FR, Dias-da-Costa JS, Olinto MTA, Pattussi MP. Prevalência e fatores associados para sobrepeso e obesidade em escolares de uma cidade no Sul do Brasil. Cad Saúde Pública 2007; 23:1361-71.

27. Terres NG, Pinheiro RT, Horta BL, Pinheiro KATP, Horta LL. Prevalência e fatores associados ao sobrepeso e à obesidade em adolescentes. Rev Saúde Pública 2006; 40:627-33.

28. Silva GAP, Balaban G, Motta MEFA. Prevalência de sobrepeso e obesidade em crianças e adolescentes de diferentes condições socioeconômicas. Rev Bras Saúde Matern Infant 2005; 5:53-9.

29. Flegal KM, Tabak CJ, Ogden CL. Overweight in children: definitions and interpretation. Health Educ Res 2006; 21:755-60.

30. Soar C, Vasconcelos FAG, Assis MAA. A relação cintura quadril e o perímetro da cintura associados ao índice de massa corporal em estudo com escolares. Cad Saúde Pública 2004; 20:1609-16. 
31. Janssen I, Katzmarzyk PT, Srinivasan SR, Chen W, Malina RM, Bouchard C, et al. Combined Influence of body mass index and waist circumference on coronary artery risk factors among children and adolescents. Pediatrics 2005; 115:1623-30.

32. Almeida CAN, Pinho AP, Ricco RG, Elias CP. Circunferência abdominal como indicador de parâmetros clínicos e laboratoriais ligados à obesidade infanto-juvenil: comparação entre duas referências. J Pediatr (Rio J.) 2007; 83:181-5.

33. Sant'Anna MSL, Tinoco ALA, Rosado LEFPL Sant'Ana LFR, Mello AC, Brito ISS, et al. Avaliação de gordura corporal pela bioimpedância elétrica $\mathrm{e}$ sua correlação com diferentes pontos anatômicos de medida da circunferência da cintura em crianças. J Pediatr (Rio J.) 2009; 85:61-6.

34. Garnett SP, Baur LA, Cowell CT. Waist-to-height ratio: a simple option for determining excess central adiposity in young people. Int J Obes Relat Metab Disord 2008; 32:1028-30.
35. Must A, Naumova EN, Phillips SM, Blum M, Dawson-Hughes B, Rand WM. Childhood overweight and maturation timing in the development of adult overweight and fatness: The Newton Girls Study and its follow-up. Pediatrics 2005; 116:620-7.

36. Adair LS, Gordon-Larsen P. Maturation timing and overweight prevalence in US adolescent girls. Am J Public Health 2001; 91:642-4.

37. Oliveira CS, Veiga GV. Estado nutricional e maturação sexual de adolescentes de uma escola pública e de uma escola privada do Município do Rio de Janeiro. Rev Nutr 2005; 18:183-91.

38. Castilho SD, Cocetti M, Azevedo-Barros Filho A. Body mass index and body composition in relation to sexual maturation. J Pediatr Endocrinol Metab 2008; 21:127-33.

39. Saito MI. Maturação sexual: auto-avaliação do adolescente. Pediatria (São Paulo) 1984; 6:111-5.

Recebido em 02/Set/2009

Versão final reapresentada em 13/Fev/2010

Aprovado em 14/Jul/2010 